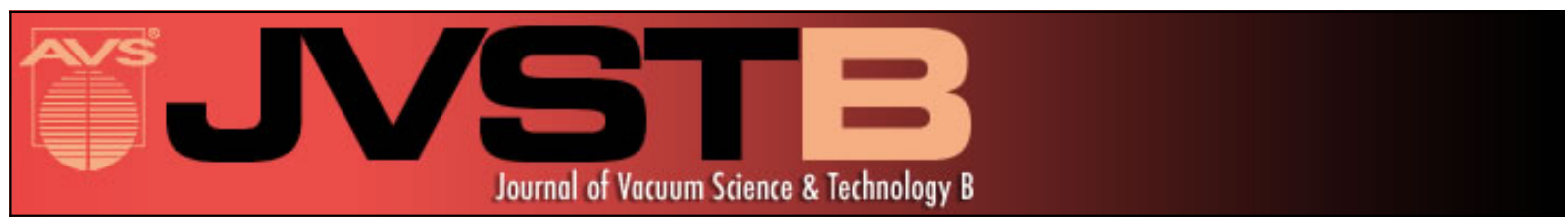

\title{
Scanning tunneling microscopy observation of local damages induced on graphite surface by ion implantation
}

L. Porte, C. H. de Villeneuve, and M. Phaner

Citation: Journal of Vacuum Science \& Technology B 9, 1064 (1991); doi: 10.1116/1.585261

View online: http://dx.doi.org/10.1116/1.585261

View Table of Contents: http://scitation.aip.org/content/avs/journal/jvstb/9/2?ver=pdfcov

Published by the AVS: Science \& Technology of Materials, Interfaces, and Processing

\section{Articles you may be interested in}

Direct observation of local chemical surface properties by scanning tunneling microscopy

AIP Conf. Proc. 1454, 9 (2012); 10.1063/1.4730676

Scanning tunneling microscopy investigation of graphite surface damage induced by goldion bombardment J. Appl. Phys. 75, 1390 (1994); 10.1063/1.356419

The effects of lowenergy ion impacts on graphite observed by scanning tunneling microscopy

J. Vac. Sci. Technol. A 9, 1261 (1991); 10.1116/1.577609

Scanning tunneling microscopy of various graphitic surfaces

J. Vac. Sci. Technol. B 9, 1061 (1991); 10.1116/1.585260

Observing the varying scanning tunneling microscopy image of graphite

J. Vac. Sci. Technol. A 8, 508 (1990); 10.1116/1.576376

\section{Instruments for Advanced Science}

Contact Hiden Analytical for further details:

w www.HidenAnalytical.com

E info@hiden.co.uk

CLICK TO VIEW our product catalogue

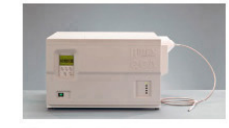

Gas Analysis

, dynamic measurement of reaction gas streams cataysis and thermal analys

molecular beam studies

fermentation, environmental and ecological studies

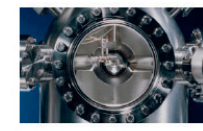

Surface Science

UHVTPD

SIN

end point detection in ion beam etch elemental imaging - surface mapping

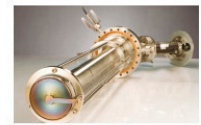

Plasma Diagnostics plasma source characterization etch and deposition process reaction

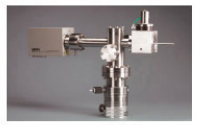

Vacuum Analysis partial pressure measurement and control of process gas er process control - vacuum diagnostics - vacuum coating process monitoring 


\title{
Scanning tunneling microscopy observation of local damages induced on graphite surface by ion implantation
}

\author{
L. Porte, C.H. de Villeneuve, and M. Phaner \\ Ecole Centrale de Lyon, CNRS-URA 404, B.P.163, 69131, Ecully, France
}

(Received 24July 1990; accepted 4 September 1990)

\begin{abstract}
Highly oriented pyrolitic graphite was implanted with $50 \mathrm{keV}$ argon ions. Low ion doses were deposited in order to observe, with the scanning tunneling microscope, surface damages due to single-ion impacts. Jon impacts were revealed by the formation of hillocks on the graphite surface. The raising of the surface is attributed to internal stresses which develop in the volume surrounding the ion track as damages and lattice defects are created by the collision cascade process. Structural transformations are induced by ion implantation: On the hillock the graphite structure was lost and no atomic organization of the surface was evidenced. Surface reconstructions and superstructures were also imaged next to some hillocks.
\end{abstract}

\section{INTRODUCTION}

The implantation of ions allows the introduction of foreign species into host materials. Because of the high kinetic energy of insertion it may be possible to modify the physical and/or chemical properties near of the solid surface and thus to create a new material with a specified property. The primary effect of ion implantation is that it creates lattice damages. The incident ions initiate collision cascades into the solid which result in interstitial and vacancy damages all along the ion track. The scanning tunneling microscope (STM) has proven to be a good tool for examination of defects created by ion implantation on the surface of solids. The effects of ion impacts have been imaged using the STM for silicon, ${ }^{1,2}$ lead sulfide, ${ }^{3}$ or graphite ${ }^{4-6}$ surfaces. Ion implantation into semiconductors received considerable attention because of its use in the fabrication of microelectronic devices. Numerous efforts have also been devoted to the implantation into graphite because this material is a good candidate for the first cladding wall of fission nuclear reactors.

One motivation for this work was to better understand the ion implantation process and the way it modifies the host materials. This paper presents a STM study relative to the first step in the modification of a graphite surface on which argon ions of $50 \mathrm{keV}$ kinetic energy have been implanted. Very low ion doses $\left(\sim 10^{11}\right.$ ions $\left.\mathrm{cm}^{-2}\right)$ were used in order to isolate single-ion impacts and to characterize the damages due to one ion impact on the surface. Implantations were performed on highly oriented pyrolitic graphite (HOPG) whose lamellar structure allows easy cleavage and preparation of large flat terraces extending over hundreds of nanometers. Such flat standard surfaces are well suited to study the effects of ion bombardment.

\section{EXPERIMENT}

The HOPG substrate was cleaved just prior to its introduction into the vacuum target chamber of an ion implanter where argon ion doses are implanted. In normal applications the target is at the center of a Faraday cup to measure the ion beam current. A diaphragm in the Faraday cup allows the beam to reach the target. In order to avoid possible contamination of the graphite surface by secondary ions the Faraday cup was removed, at the expense of the current measure.
In these conditions several ion doses in the range $10^{11}-10^{16}$ ions $\mathrm{cm}^{-2}$ were implanted into polycrystalline graphite and the exact quantities of argon atoms deposited were determined for highest fluencies by Rutherford backscattering spectroscopy (RBS) to establish the calibration curve for implantation. In addition RBS allowed to verify that no contaminant was introduced during the implantation process. This was confirmed by an analysis of the HOPG surface using $x$-ray photoelectron spectroscopy (XPS). The only contaminant detectable by XPS was oxygen. For the low ion doses which are of prime concern here $\left(10^{11}-10^{12}\right.$ ions $\mathrm{cm}^{-2}$ ) the quantity of oxygen was very low and similar to the surface oxygen present on the nonimplanted HOPG surface.

After implantation the surface was observed using the STM in air. Imaging was carried out in the constant current mode, with scanning speeds of 1 or $2 \mathrm{~Hz}$ and Pt-Ir electrochemically etched tips. Tip voltage and tunneling current were fixed at $+100 \mathrm{mV}$ and $1 \mathrm{nA}$, respectively. Local barrier-height images were also recorded simultaneously with the constant current topographic image. The tip-surface distance was modulated at $2 \mathrm{kHz}$ frequency with a peak to peak amplitude of $0.15 \mathrm{~nm}$. As it is commonly reported for measurements in air ${ }^{7}$ very low values of the barrier height (less than $1 \mathrm{eV}$ ) were obtained.

\section{RESULTS AND DISCUSSION}

Figure 1(a) shows a typical STM image of the HOPG surface after implantation of a low ion dose $\left(5 \times 10^{\text {it }}\right.$ ions $\mathrm{cm}^{-2}$ ). The initially flat surface has been modified by implantation and five hillocks are visible on this $30 \times 30 \mathrm{~nm}^{2}$ image. We verified on graphite implanted at ion doses ranging between $10^{11}$ and $10^{13}$ ions $\mathrm{cm}^{-2}$ that a statistical one to one correlation exists between the number of hillocks and the number of implanted ions. ${ }^{4}$ For doses greater than $10^{1,3}$ ions $\mathrm{cm}^{-2}$ hillocks begin to cover the whole of the surface and cannot be counted. Thus each hillock is identified as the physical signature of the implantation of one individual argon ion into graphite.

In the constant current mode of imaging used, the $z$-tip displacement should reproduce the surface topography provided there is no change in the density of states and local 


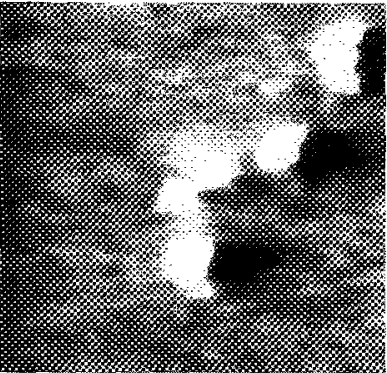

(a)

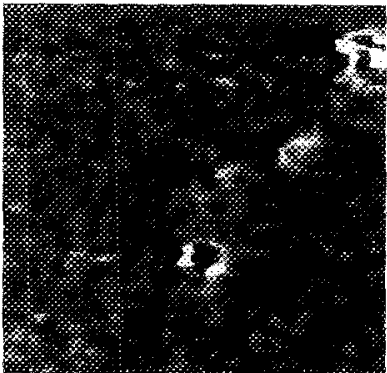

(b)
FIG. 1. STM image of a $30 \times 30 \mathrm{~nm}^{2}$ area of HOPG implanted by $\mathrm{Ar}^{+}$of 50 $\mathrm{keV}$ kinetic energy at a dose of $5 \times 10^{11}$ ions $\mathrm{cm}^{2}$. (a) Topographic image showing five hiliocks. $\Delta Z=2 \mathrm{~nm}$ from black bottom to white top. (b) Simultaneous recorded barrier height image $(d \ln I / d z)$.

work function on the surface. Changing the tunneling conditions did not alter significantly the recorded images. Hillocks were obtained whatever the voltage and polarity of the tip. Images of local barrier height are known to contain the contribution from the work function plus some geometric contribution. This latter contribution comes when the angle between the direction of modulation $(z)$ and the surface changes. ${ }^{8}$ Figure 1 compares (a) the topographic image and (b) the local barrier height image which were recorded simultaneously. The main effect visible on the barrier height image is an increase of the noise for highest hillocks. This can be attributed to a mechanical excitation of the tip when it crosses the hillock. Indeed it has been shown that for STM imaging in air tip-surface mechanical contact via a contamination layer modify the behavior of the tunnel interface. ${ }^{7}$ The barrier height measurements we have performed so far do not prove that some work function change takes place on the hillock and it seems very reasonable to first consider these hillocks as the manifestation of the crystalline modiffcation of the surface.

The hillocks have variable height ranging from one to several tenths of nanoneter. Their lateral extension is also variable and can reach a few nanometers. The formation of a bump at the ion impact may be interpreted as the result of the strong perturbation which takes place into the sample all around the track of the impinging ion. The primary ion which penetrates into the solid with high velocity generates a collision cascade phenomenon. Collision cascades are well modelled using calculation of the Monte Carlo type such as the TRIM code calculation. ${ }^{9}$ This statistical nature of the cascade process is reflected by the differences in the size of hillocks. The parameters of the cascade obtained by TRIM code calculation result from a statistic over thousands of penetrating ions. For argon of $50 \mathrm{keV}$ into graphite, the calculation gives an average depth of penetration of $42 \mathrm{~nm}$ with a standard deviation of $12 \mathrm{~nm}^{4}{ }^{4}$ Vacancies and intersticials are created along the volume concerned by the collision cascade and if the deformation of the surface reflects the damage in this volume one can imagine that different deformations will occur according to each individual path of the penetrating ion.

Figures 2-4 show the impact of individual ions imaged at high magnification. These images illustrate the differences which can occur on the damaged surface after the ion im-
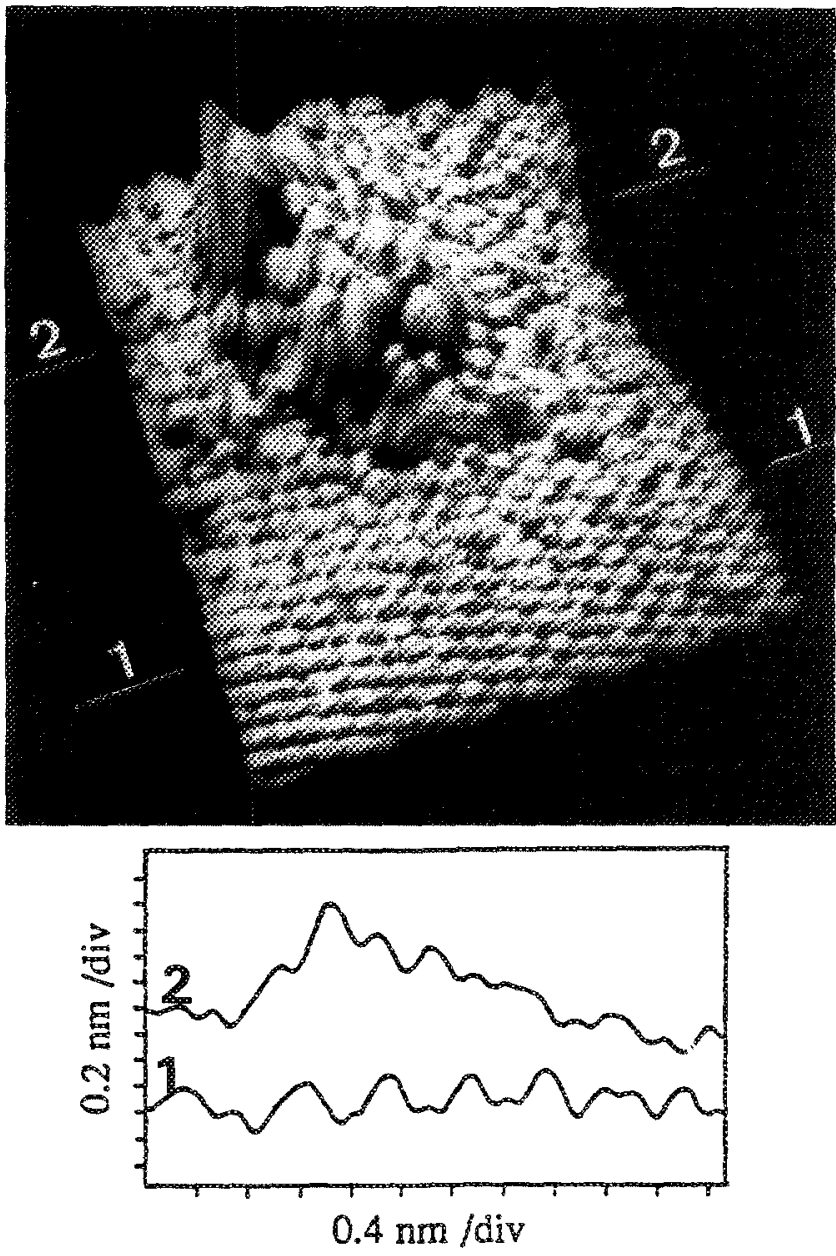

FIG. 2. STM image of an implanted HOPG sample showing lattice damages due to the impact of one Ar ${ }^{+}$of $50 \mathrm{keV}$ kinetic energy. Area $=4.5 \mathrm{~nm} \times 6$ nm.

pact. The typical image of a hillock which has raised on the unpertubed graphite lattice is exemplified by the Figs. 2 and 3 details the hillock itself. Here the graphite lattice appears distorted as it rises from right to left until the surface layer breaks and a step of about $1 \mathrm{~nm}$ is formed. In the atomic rearrangement following the collision cascade vacancy dislocation loops and extra planes of intersticials can form. Such processes would develop interplanar stresses in the volume concerned by the collision cascade and a subsequent raising of the surface at the impact point. This hypothesis is in agreement with the well-documented macroscopic dimensional change of graphite crystals upon irradiation. ${ }^{10} \mathrm{At}$ room temperature defects created into graphite by irradiation induce an expansion of the crystal along the $c$-axis direction. Then the hillocks observed would be the first manifestation of macroscopic dimensional changes. Deep damages develop a strength which deforms the surface graphite layer. Occasionally the elastic limit can be overstepped and the surface breaks, making a step as shown in Fig. 3. Hillocks of variable high, ranging from one to some tenths of nm, have been imaged. ${ }^{4}$ One can remark that the hillock in Fig. 3 was one of the most elevated we have found and that steps formed around hillocks were very rarely imaged. 

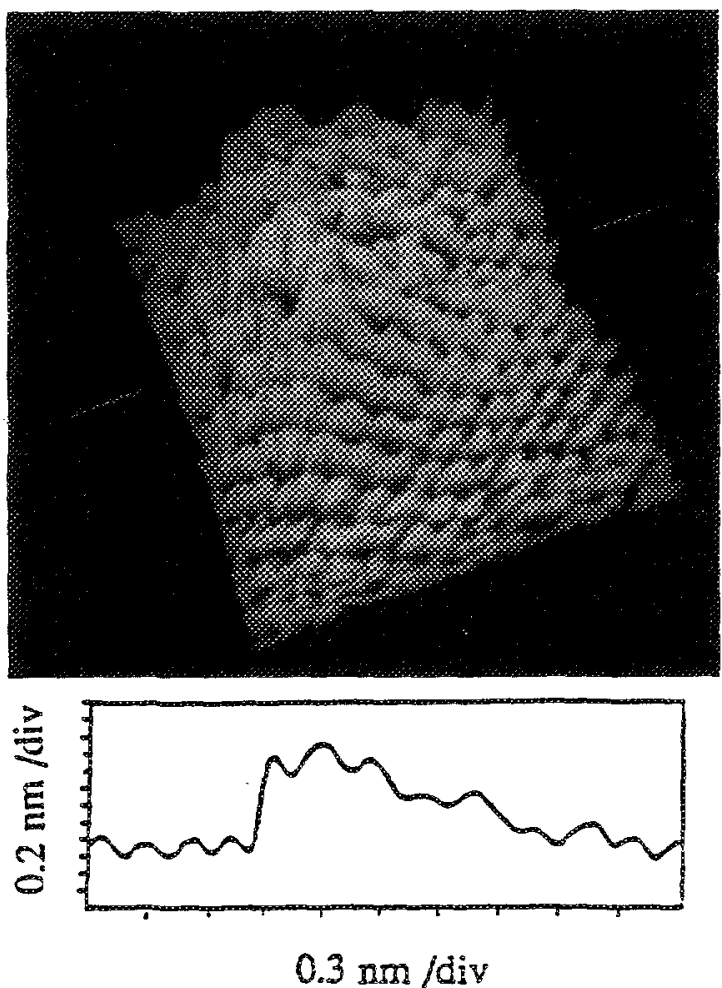

1G. 3. Distortion of the graphite lattice on the hillock formed at Ar impact of $50 \mathrm{keV}$ kinetic energy. Area $=3 \mathrm{~nm} \times 4 \mathrm{~nm}$.

An other kind of interesting defect is shown in Fig. 4. Apart the normal graphite structure a superstructure appears near of the ion impact, around the higher part of the hillock which does not exhibit any regular structure as it is frequently observed. The unit cell of this superstructure appears hexagonal with a lattice parameter of $0.38 \mathrm{~nm}$. Marchon et al. have measured structures with dimensions $0.27 \times 0.45 \mathrm{~nm}^{2}$ on the surface of amorphous carbon films. ${ }^{11}$ They tentatively attributed them to the $1 \times 2$ reconstruction of the nonhydrogenated diamond surface. ${ }^{12}$ Because of their great technological interest the bonding in amorphous carbon fiims has attracted considerable attention. The properties of disordered forms of carbon are generally interpreted by the model of a metastable phase consisting of a mixture of tetrahedral $\left(s p^{3}\right)$ and trigonal $\left(s p^{2}\right)$ bonding in the matrix. On the other hand strong evidences ${ }^{13,14}$ exist for a link between the development of tetrahedral bonding and the ion bombardment used in the preparation of amorphous fims. It has also been demonstrated from resistivity measurements that the same state is achieved whether one adds diamond bonds to graphite, by evaporation or sputtering of graphite, or generates graphite bonds in diamond by implantation. ${ }^{15}$ In other words a mixture of $s p^{3}$ and $s p^{2}$ bonds could be found in disordered carbon irrespective of the preparation method. Diamondlike bonds are expected to occur in amorphous carbon as in implanted graphite. However the graphite (0001) and the diamond (111) surfaces have a good matching. This make difficult to attribute the superstructure in Fig. 4 to the diamond (111) $1 \times 2$ reconstructed surface, as Marchon et al. did for amorphous carbon film, because one could not understand in that case the disorientation visible between
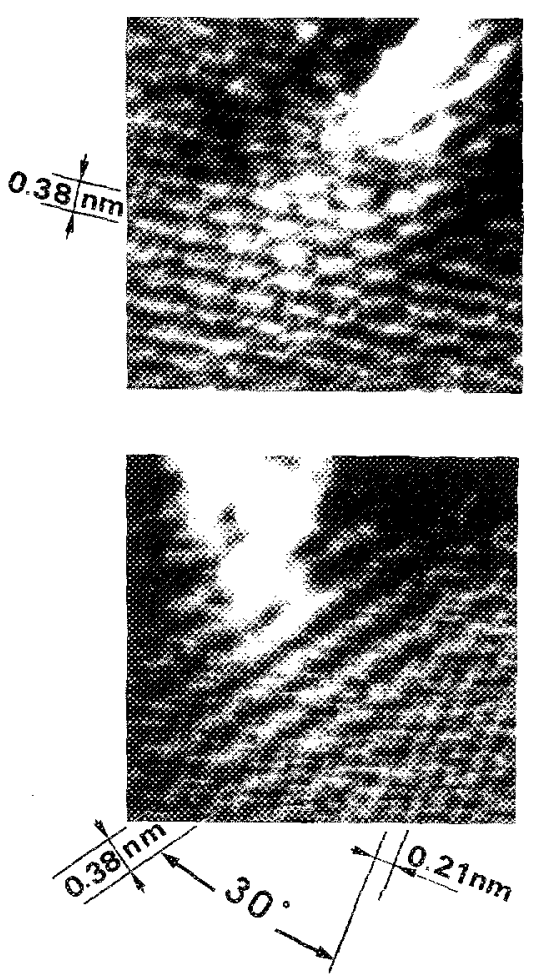

FIG. 4. STM images of superstructures created at around hillock due to A ${ }^{\prime}$ impact of $50 \mathrm{keV}$ kinetic energy.

the superstructure and the graphite lattice. In addition Raman studies of graphite implanted by high energy ions do not exhibit the $1332 \mathrm{~cm}^{-1}$ line from diamond but a line at $\sim 1360 \mathrm{~cm}{ }^{1}$ which appears to be characteristic of disorder in graphite. ${ }^{16,17}$ We have verified that the $1360 \mathrm{~cm}^{-1} \mathrm{Ra}-$ man ine, which is absent for virgin HOPG, appeared after the implantation of $\sim 10^{11}$ argon ions $\mathrm{cm}^{-2}$. This Raman line was also observed by Rabe et al ${ }^{18}$ for stepped HOPG surfaces, in connection with the presence of a $\sqrt{3} \times \sqrt{3} R 30^{\circ}$ superstructure which appeared on STM images. This superstructure was localized near point defects and steps. The superstructure in Fig. 4 is also rotated from the lattice graphite by about $30^{\circ}$ and the measured lattice parameter is 0.38 $\mathrm{nm}$. This is very similar to Rabe et al.'s experiment who found a lattice parameter of $0.37 \mathrm{~nm}$. Then this superstructure manifests when the perfect $2 \mathrm{D}$ graphite lattice experiences some perturbation. Next to the hillock the reconstruction of the surface may be interpreted as the accommodation of the graphite lattice to the stress developed in the volume modified by the collision cascade. A distribution of carbon bond order must be taken into account to explain the properties of hard carbon films. Weissmantel et al. ${ }^{19}$ proposed a structural model for disordered carbon consisting of puckered $n$-fold carbon rings ( $n=3-8$ ) with tetrahedral crosslinks. Then one would like to get more evidence for the presence of $s p^{3}$ bonds in disordered carbon phase, such as implanted graphite. So far we have been unable to resolve the atomic structure within the small protusion which forms the superstructure. One can however estimate the lateral size of these protusions to $0.24 \mathrm{~nm}$ and notice that their apparent morphology and size are compatible with the tetrahedral 
unit mesh of the diamond (111) surface which is $0.252 \mathrm{~nm}$ on side. One final remark concerns the rapid intensity decay of the reconstruction which vanishes over a range of one or two nanometers from the hillock. This behavior could be tentatively interpreted within the concept of hypersonic shock waves created during a collision cascade. ${ }^{20}$ Molecular dynamic computer simulations support the idea that shock front-type process propagate away from the ion's track in the near-surface region. ${ }^{21}$ It is tempting to suggest, in the course of this concept, that the deformation and reconstruction of the graphite surface around the ion impact are the memory effect of a shock-wave process.

\section{CONCLUSIONS}

Low doses of argon ions-fiuence $\sim 10^{11}$ ions $\mathrm{cm}^{-2}$, kinetic energy $=50 \mathrm{keV}$ - have been implanted on cleaved HOPG surfaces. The dose was chosen in order to isolate the effect of a single-ion impact. The imaging of implanted surface using the STM reveals that small hillocks form on the graphite surface at the impact of individual ions. The raising of the surface is attributed to interplanar stresses which are generated along the ion track after the collision cascade process. It is interpreted as a first manifestation of the macroscopic expansion of graphite crystals which are known to expand along the $c$-axis direction upon irradiation. These hillocks have measured height in the tenth of nanometer range and lateral size in the nanometer range. The differences in size reflect the statistical rature of the collision cascade process. Generally the higher part of hillocks does not exhibit any organized structure. However superstructures can occasionally appear around the hillock, in addition to the normal graphite structure. Ion implantation induces phase transformation into graphite and the possible connection of these superstructures with the formation of new carbon phases and $s p^{3}$-like tetrahedral bonds is discussed.

\section{ACKNOWLEDGMENTS}

This work was supported by the Direction des Recherches Etudes et Techniques under Contract No. 89/172 DRET. We would like to thank $F$. Thibaudau and $C$. Mouttet who generously gave us their original imaging software.

'I. H. Wison, N. J. Zheng, U. Xnipping, and I. S. T. Tsong, Phys. Rev. B 38,8444 (1988)

${ }^{2}$ R. M. Feenstra and G. S. Oehriein, Appl. Phys. Lett. 47, 97 (1985).

'I. H. Wilson, N. J. Zheng, U. Knipping and I. S. T. Tsong, Appl. Phys. Lett. 53, 2039 (1988).

${ }^{4}$ I. Porte, M. Phaner, C. H. de Villeneuve, N. Moncoffre, and 3. Tousset, Nucl. Instrum. Meth. Phys. Res. B 44, 116 (1989).

${ }^{5}$ R. Coratger, A. Claverie, F. Ajustron, and J. Beatuvillain, Surf. Sci. 227, 7 (1990).

${ }^{6}$ Y. Koga, Y. Miyazaki, and N. Nakagini, Jpñ. J. Appl. I'hys. 27, 1.976 (1988).

'H.J. Mamin, E. Ganz, D. W. Abraham, R. F. Thompson, and J. Clarke, Phys. Rev, B 3\&, 9015 (1986).

' J. M. Gomez-Rodriguez, J. Gomez-Herrero, and A. M. Haro, Surf. Sci $220,152(1989)$.

${ }^{9}$ J. F. Ziegler, J. P. Biersack, and Y. Littmark, in The Stopping and Range of Ions in Solids, edited by 3. F. Tiegler (Pergamon, New York, 1975), Vol. 1.

${ }^{10}$ B. T. Kelly, Physics of Graphite (Applied Science Publication London, 1981).

${ }^{11}$ W. Marchon, M. Salmeron, and W. Siekhaus, Phys. Rev. B 39, 12907 (1989).

${ }^{12}$ B. B. Pate, Surf, Sci. 165, 83 (1986).

${ }^{12}$ C. Weissmantel, J. Yac. Sci, Technol. 18, $179(1981)$.

${ }^{14}$ N. Savvides, I. Appl. Phys. 59, 4133 (1986).

'5. J. Hauser and J. R. Patel, Solid State Commun. 18, 789 (1976).

${ }^{15}$ F. Tuinstra and J. L. Koenig, J. Chem. Phys. 53, 1126 (1970)

${ }^{17}$ B. S. Elman, M. S. Dressethaus, G. Dressehaus, E. W. Maby, and H. Mazurek, Phys. Rev. 24, 1027 (1981).

${ }^{18}$ J. P. Rabe, M. Sano, D. Batchelder, and A. A. Kalatchev, J. Microsc. 152, $573(1988)$.

${ }^{19} \mathrm{G}$. Weissmantel, K. Bewilogua, K. Brever, D. Dietrich, U. Ebershach, H. J. Erler, B. Rau, and G. Reisse, Thin Solid Films 96, 31 (1982).

${ }^{20} \mathrm{~K}$. B. Winterbon, Radiat. Eff. Lett. 57,89 (1980).

${ }^{21}$ R. P. Webb and D. F. Harrison, Jr., Appl. Phys. Lett. 39, 311 (1981). 